\title{
Sub-Quantum Fisher Information
}

\author{
M. Cerezo, ${ }^{1,2,3, *}$ Akira Sone, ${ }^{1,2,3,4, *}$ Jacob L. Beckey, ${ }^{1,5,6}$ and Patrick J. Coles ${ }^{1,3}$ \\ ${ }^{1}$ Theoretical Division, Los Alamos National Laboratory, Los Alamos, New Mexico 87545, USA \\ ${ }^{2}$ Center for Nonlinear Studies, Los Alamos National Laboratory, Los Alamos, New Mexico 87545, USA \\ ${ }^{3}$ Quantum Science Center, Oak Ridge, TN 37931, USA \\ ${ }^{4}$ Aliro Technologies, Inc. Boston, Massachusetts 02135, USA \\ ${ }^{5}$ JILA, NIST and University of Colorado, Boulder, Colorado 80309 \\ ${ }^{6}$ Department of Physics, University of Colorado, Boulder, Colorado 80309
}

\begin{abstract}
The Quantum Fisher Information (QFI) plays a crucial role in quantum information theory and in many practical applications such as quantum metrology. However, computing the QFI is generally a computationally demanding task. In this work we analyze a lower bound on the QFI which we call the sub-Quantum Fisher Information (sub-QFI). The bound can be efficiently estimated on a quantum computer for an $n$-qubit state using $2 n$ qubits. The sub-QFI is based on the super-fidelity, an upper bound on Uhlmann's fidelity. We analyze the sub-QFI in the context of unitary families, where we derive several crucial properties including its geometrical interpretation. In particular, we prove that the QFI and the sub-QFI are maximized for the same optimal state, which implies that the sub-QFI is faithful to the QFI in the sense that both quantities share the same global extrema. Based on this faithfulness, the sub-QFI acts as an efficiently computable surrogate for the QFI for quantum sensing and quantum metrology applications. Finally, we provide additional meaning to the sub-QFI as a measure of coherence, asymmetry, and purity loss.
\end{abstract}

The Quantum Fisher Information (QFI) is a fundamental quantity in quantum information theory [1-8] with applications to quantum metrology [9-13], quantum channel estimation [14], and condensed matter physics [15-17]. Given a quantum state $\rho_{\theta}$ parameterized by a parameter $\theta$, the estimation uncertainty of $\theta$ is lower bounded by the inverse of the QFI $I\left(\rho_{\theta}\right)$ via the quantum Cramér-Rao bound. For instance, in a quantum metrology setting, the larger the QFI is, the more information about $\theta$ one can obtain from $\rho_{\theta}$. This indicates that the QFI can be regarded as a good measure to evaluate the sensing capabilities of a quantum state.

Despite its tremendous relevance, computing the QFI is usually a challenging task for both classical and quantum computers [18]. Most classical methods that estimate $I\left(\rho_{\theta}\right)$ require: 1$)$ full knowledge of $\rho_{\theta}$, usually obtained via full quantum state tomography, and 2) diagonalization of density matrices [19]. However, both of these tasks are inefficient for large problem sizes because of the exponential scaling of the Hilbert space dimension. In addition, even if one can access $\rho_{\theta}$ on a quantum computer, no quantum algorithm can efficiently, directly compute the QFI due to its non-linear functional dependence on $\rho_{\theta} \quad[20]$.

One can potentially circumvent the issue of exactly evaluating the QFI by instead estimating efficiently computable bounds on $I\left(\rho_{\theta}\right)$, as the latter provides a range in which the QFI actually lies [21-26]. For metrology applications (where higher QFI is better), lower bounds are of particular interest as they can guarantee that a state $\rho_{\theta}$ has at least a certain QFI value. This can be used to check if the quality of the state for quantum sensing is above a desired threshold.

\footnotetext{
* The first two authors contributed equally to this work.
}

Recently, the advent of the so-called Noisy Intermediate Scale Quantum (NISQ) computers [27] has opened the exciting possibility of using these near-term devices for quantum sensing and quantum metrology tasks. Among the most promising applications using NISQ devices are variational quantum approaches [28-32] where one attempts to variationally prepare the state that maximizes the QFI [33-37]. Indeed, most of these algorithms optimize lower bounds on the QFI by training a unitary that prepares the best possible input state.

In this work, we analyze a lower bound for the QFI, which we call the sub-Quantum Fisher Information (subQFI), and which can be efficiently computed on a quantum computer for an $n$ qubit state $\rho_{\theta}$ with $2 n$ qubits. The properties of sub-QFI and its operational meaning are studied for states belonging to the unitary families.

Our main results are as follows. First, we obtain a simple analytical expression for the sub-QFI for unitary families which allows us to analyze its algebraic properties and its geometric interpretation. Second, we rigorously prove that the optimal mixed state that maximizes the QFI is always the same optimal state that maximizes the sub-QFI. This allows us to conclude that the sub-QFI is faithful to the QFI in the sense that both quantities share the same global extrema. Then, we relate the sub-QFI to other relevant quantities in the literature to connect the sub-QFI with coherence and asymmetry measures, and to the fragility of the probe state to stochastic error in metrology tasks.

Finally, we discuss using the sub-QFI as an efficiently computable surrogate for the QFI for quantum metrology applications in NISQ devices. Particularly, the fact that the sub-QFI can be efficiently computed with just $2 n$ qubits makes it an attractive quantity. Moreover, the faithfulness of the sub-QFI guarantees that if one maximizes this quantity, then the state prepared will also max- 
imize the QFI. Hence, we expect that our contributions can be applied in the near-future for various practical metrology tasks using near-term quantum computers.

Sub-Quantum Fisher Information. Let $\rho$ be an $n$-qubit input state, known as the probe state, which interacts with a source that encodes the information of a parameter $\theta$ of interest. The state resulting from the interaction, $\rho_{\theta}$, is known as the exact state. The ultimate precision $\Delta \theta$ in estimating $\theta$ from $\nu$ measurements on $\rho_{\theta}$ is bounded by the Quantum Fisher Information (QFI) $I\left(\rho_{\theta}\right)$ via the quantum Cramér-Rao bound:

$$
(\Delta \theta)^{2} \geqslant \frac{1}{\nu I\left(\rho_{\theta}\right)} .
$$

The QFI is defined as

$$
I\left(\rho_{\theta}\right)=\operatorname{Tr}\left[L_{\theta}^{2} \rho_{\theta}\right]
$$

where $L_{\theta}$ is the Symmetric Logarithmic Derivative (SLD) operator satisfying $\partial_{\theta} \rho_{\theta}=\frac{1}{2}\left(L_{\theta} \rho_{\theta}+\rho_{\theta} L_{\theta}\right)$. Moreover, defining the error state, $\rho_{\theta+\delta}$, the QFI can also be related to Uhlmann's quantum fidelity from the equality [38-40]

$$
\begin{aligned}
I\left(\rho_{\theta}\right) & =-4 \lim _{\delta \rightarrow 0} \partial_{\delta}^{2} F\left(\rho_{\theta}, \rho_{\theta+\delta}\right) \\
& =8 \lim _{\delta \rightarrow 0} \frac{1-F\left(\rho_{\theta}, \rho_{\theta+\delta}\right)}{\delta^{2}},
\end{aligned}
$$

where $F(\rho, \sigma)=\|\sqrt{\rho} \sqrt{\sigma}\|_{1}$ is the quantum fidelity, and $\|A\|_{1}=\operatorname{Tr}\left[\sqrt{A A^{\dagger}}\right]$ is the trace norm. Intuitively, Eq. (3) relates the QFI to how sensitive the probe state $\rho$ is to small parameter shifts, $\delta$. The greater the concavity of $F\left(\rho_{\theta}, \rho_{\theta+\delta}\right)$ (quantified by its second derivative with respect to $\delta$ ), the larger the QFI. Viewed another way, Eq. (4) has the interpretation that the more distinguishable the exact and error states are, the more information one can obtain about the unknown parameter via measurement.

Based on Eq. (4) the definition of the sub-Quantum Fisher Information (sub-QFI) is as follows.

Definition 1. Let $\rho_{\theta}$ and $\rho_{\theta+\delta}$ be the exact and error state, respectively. The sub-QFI is defined as:

$$
\mathcal{I}\left(\rho_{\theta}\right)=8 \lim _{\delta \rightarrow 0} \frac{1-\sqrt{G\left(\rho_{\theta}, \rho_{\theta+\delta}\right)}}{\delta^{2}},
$$

where for two quantum states $\rho$ and $\sigma$

$$
G(\rho, \sigma)=\operatorname{Tr}[\rho \sigma]+\sqrt{\left(1-\operatorname{Tr}\left[\rho^{2}\right]\right)\left(1-\operatorname{Tr}\left[\sigma^{2}\right]\right)},
$$

is the so-called super-fidelity.

As shown in [41], the square root of the superfidelity $[42,43]$ upper bounds Uhlmann's fidelity

$$
F\left(\rho_{\theta}, \rho_{\theta+\delta}\right) \leqslant \sqrt{G\left(\rho_{\theta}, \rho_{\theta+\delta}\right)} .
$$

Hence, combining (4), (5), and (7) we readily find that the sub-QFI is a lower bound for the QFI

$$
\mathcal{I}\left(\rho_{\theta}\right) \leqslant I\left(\rho_{\theta}\right)
$$

Equation (6) shows that each term in the sub-QFI can be efficiently estimated as there exists an algorithm that computes state overlaps of the form $\operatorname{Tr}\left[\rho_{\theta} \rho_{\theta+\delta}\right]$ with a depth-two quantum circuit and classical post-processing that scales linearly with $n$ [44]. We note that the subQFI bound has been used before in the study of quantum coherence, entanglement witnesses, and in quantum optics [45-47]. It is because of the broad utility of the sub-QFI that an in-depth analysis of its properties is of such importance.

Main results. In what follows we consider unitary families, i.e., we analyze the case when the parameter $\theta$ is encoded in the probe state $\rho$ via

$$
\rho_{\theta}=W_{\theta} \rho W_{\theta}^{\dagger} \quad \text { with } \quad W_{\theta}=e^{-i \theta H},
$$

for some $\theta$-independent Hermitian operator $H$, usually called the generator. As shown in the Appendix, the following theorems and propositions hold.

Theorem 1 (Explicit form). For unitary families, the sub-QFI of Definition 1 can be expressed as

$$
\begin{aligned}
\mathcal{I}\left(\rho_{\theta}\right) & =4\left(\operatorname{Tr}\left[\rho^{2} H^{2}\right]-\operatorname{Tr}[\rho H \rho H]\right) \\
& =-2 \operatorname{Tr}\left[[\rho, H]^{2}\right] .
\end{aligned}
$$

Note that Theorem 1 implies that $\mathcal{I}\left(\rho_{\theta}\right)=\mathcal{I}(\rho)$ since $\left[W_{\theta}, H\right]=0$, meaning that the sub-QFI is independent of $\theta$. However, we continue to use the notation $\mathcal{I}(\rho)$ for consistency. Given the spectral decomposition $\rho=$ $\sum_{j=1}^{r} \lambda_{j}\left|\lambda_{j}\right\rangle\left\langle\lambda_{j}\right|$, with $r=\operatorname{rank}(\rho)$ and with $\lambda_{i} \geqslant \lambda_{i+1}$, we find from Eq. (10) that

$$
\mathcal{I}(\rho)=2 \sum_{i, j=1}^{r}\left(\lambda_{i}-\lambda_{j}\right)^{2}\left|\left\langle\lambda_{i}|H| \lambda_{j}\right\rangle\right|^{2}
$$

The following properties of the sub-QFI can be readily derived from [48]:

- Non-negativity: $0 \leqslant \mathcal{I}(\rho)$.

- Convexity: $\mathcal{I}\left(\sum_{k} \omega_{k} \rho^{(k)}\right) \leqslant \sum_{k} \omega_{k} \mathcal{I}\left(\rho^{(k)}\right)$ for arbitrary quantum states $\rho^{(k)}$ and for positive coefficients $\omega_{k}$ such that $\sum_{k} \omega_{k}=1$.

- Modified additivity: Given a bipartite quantum system $\mathcal{H}_{A} \otimes \mathcal{H}_{B}$, let $\rho_{\theta}^{A}\left(\rho_{\theta}^{B}\right)$ and $H_{A}\left(H_{B}\right)$ respectively be a quantum state and a Hermitian operator on $\mathcal{H}_{A}\left(\mathcal{H}_{B}\right)$. Then, for $\rho_{\theta}=$ $\rho_{\theta}^{A} \otimes \rho_{\theta}^{B}$ and $H=H_{A} \otimes \mathbb{1}+\mathbb{1} \otimes H_{B}$, we have $\mathcal{I}\left(\rho_{\theta}\right)=\operatorname{Tr}\left[\left(\rho_{\theta}^{A}\right)^{2}\right] \mathcal{I}_{A}\left(\rho_{\theta}^{A}\right)+\operatorname{Tr}\left[\left(\rho_{\theta}^{B}\right)^{2}\right] \mathcal{I}_{B}\left(\rho_{\theta}^{B}\right)$ where $\mathcal{I}_{A(B)}\left(\rho_{\theta}^{A(B)}\right)=-2 \operatorname{Tr}\left[\left[\rho_{\theta}^{A(B)}, H_{A(B)}\right]^{2}\right]$. 
- Modified decreasing under the partial trace: Given a bipartite quantum system $\mathcal{H}=\mathcal{H}_{A} \otimes \mathcal{H}_{B}$ and a state $\rho_{\theta} \in \mathcal{H}$, let $\rho_{\theta}^{A}=\operatorname{Tr}_{B}\left[\rho_{\theta}\right]$ denote the reduced state on subsystem $A$. Then, for $H=H_{A} \otimes \mathbb{1}$ with $H_{A}$ a Hermitian operator in $\mathcal{H}_{A}$, we have $\mathcal{I}\left(\rho_{\theta}\right) \geqslant$ $\mathcal{I}_{A}\left(\rho_{\theta}^{A}\right) / d_{B}$, where $d_{B}$ is the dimension of $\mathcal{H}_{B}$.

Now, let us define an operator $\Lambda_{\theta}$ for a full rank probe state $\rho$,

$$
\Lambda_{\theta}=\left(\partial_{\theta} \rho_{\theta}\right) \rho_{\theta}^{-1}, \quad \Lambda_{\theta}^{\dagger}=\rho_{\theta}^{-1} \partial_{\theta} \rho_{\theta},
$$

which plays a role of a non-Hermitian SLD (nSLD) operator [49] since $\partial_{\theta} \rho_{\theta}=\frac{1}{2}\left(\Lambda_{\theta} \rho_{\theta}+\rho_{\theta} \Lambda_{\theta}^{\dagger}\right)$ and $\operatorname{Tr}\left[\Lambda_{\theta} \rho_{\theta}\right]=$ $\operatorname{Tr}\left[\rho_{\theta} \Lambda_{\theta}^{\dagger}\right]=0$. Then, we can obtain the following result.

Proposition 1 (Non-Hermitian SLD operator). For full rank probe states $\rho$, the sub-QFI can be expressed as

$$
\mathcal{I}\left(\rho_{\theta}\right)=2 \operatorname{Tr}\left[\Lambda_{\theta}^{\dagger} \Lambda_{\theta} \rho_{\theta}^{2}\right]
$$

where $\Lambda_{\theta}$ is the nSLD operator in (13).

If $\left[\rho_{\theta}, \partial_{\theta} \rho_{\theta}\right]=0$, then $\left[\rho_{\theta}^{-1}, \partial_{\theta} \rho_{\theta}\right]=0, \Lambda_{\theta}$ becomes the standard SLD operator $L_{\theta}$ in Eq. (2) [50, 51]. Note that when $\rho_{\theta}$ is a pure state $\left|\psi_{\theta}\right\rangle\left\langle\psi_{\theta}\right|$, then Eq. (8) is saturated because the super-fidelity becomes the standard fidelity when $\rho_{\theta}$ is a pure state. In general, however, the sub-QFI will only lower bound the QFI. Nonetheless, one can still derive the following theorem which shows that $\mathcal{I}(\rho)$ and $I(\rho)$ are always simultaneously maximized for the same optimal state.

Theorem 2 (Optimal state). For any quantum state $\rho$ and for any Hermitian generator $H$, the sub-QFI of Definition 1 and the QFI in (4) are maximized for the same state preparation $U \rho U^{\dagger}$, with the optimal unitary being

$$
U_{*}=\underset{U}{\arg \max } \mathcal{I}\left(U \rho U^{\dagger}\right)=\underset{U}{\arg \max } I\left(U \rho U^{\dagger}\right) .
$$

Here, the maximum is taken over the unitary group of degree $d$, with $d=2^{n}$.

Theorem 2 shows that the sub-QFI and the QFI share the same optimal state $\rho^{*}=U_{*} \rho U_{*}^{\dagger}$. Hence, as shown in Fig. 1, sub-QFI and the QFI are simultaneously maximized by the input state $\rho^{*}$. Moreover, since $\mathcal{I}\left(\rho_{\theta}\right)=$ $0 \leftrightarrow I\left(\rho_{\theta}\right)=0$ (as seen from (12)), then Theorem 2 implies the sub-QFI is faithful to the QFI, in the sense that both QFI and sub-QFI share the same global extrema.

Let $\left\{h_{j}\right\}$ and $\left\{\left|h_{j}\right\rangle\right\}$ respectively be the set of eigenvalues and associated eigenvectors of $H$ (with $h_{j} \geqslant h_{j+1}$ ). Then, as shown in [52] the state that maximizes the QFI, and hence the sub-QFI, is given by $\rho^{*}=\sum_{j=1}^{d} \lambda_{j}\left|\phi_{j}\right\rangle\left\langle\phi_{j}\right|$, where

$$
\left|\phi_{j}\right\rangle= \begin{cases}\frac{1}{\sqrt{2}}\left|h_{j}\right\rangle+\frac{1}{\sqrt{2}} e^{i \chi}\left|h_{d-j+1}\right\rangle & (2 j<d+1) \\ \left|h_{j}\right\rangle & (2 j=d+1) \\ \frac{1}{\sqrt{2}}\left|h_{j}\right\rangle-\frac{1}{\sqrt{2}} e^{i \chi}\left|h_{d-j+1}\right\rangle & (2 j>d+1)\end{cases}
$$

and where $\chi \in \mathbb{R}$ is an arbitrary phase. Then, the following corollary holds.

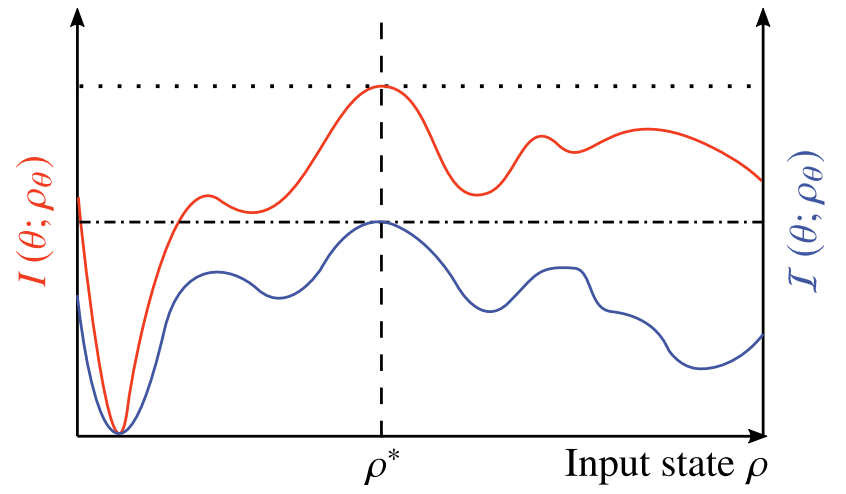

FIG. 1. Schematic diagram of the QFI and sub-QFI versus the input state $\rho$. The sub-QFI $\mathcal{I}\left(\rho_{\theta}\right)$ is a lower bound for the QFI $I\left(\rho_{\theta}\right)$. As shown by Theorem 2, the QFI and sub-QFI reach their maximum for the same optimal input state. Since $\mathcal{I}\left(\rho_{\theta}\right)=0 \leftrightarrow I\left(\rho_{\theta}\right)=0$, the sub-QFI is faithful to the QFI in the sense that both QFI and sub-QFI share the same global extrema.

Corollary 1 (Maximal sub-QFI). The maximal sub-QFI with respect to all $d \times d$ state preparation unitaries is

$$
\begin{aligned}
\mathcal{I}\left(\rho^{*}\right) & =\max _{U} \mathcal{I}\left(U \rho U^{\dagger}\right) \\
& =\frac{1}{2} \sum_{k=1}^{d}\left(\lambda_{k}-\lambda_{d-k+1}\right)^{2}\left(h_{k}-h_{d-k+1}\right)^{2} .
\end{aligned}
$$

Finally, the following proposition provides a geometrical interpretation to the sub-QFI.

Proposition 2 (Geometrical interpretation). The subQFI can be expressed as

$$
\mathcal{I}\left(\rho_{\theta}\right)=\left.\partial_{\theta}^{2} D_{H S}\left(\rho, \rho_{\theta}\right)\right|_{\theta=0},
$$

where $D_{H S}(A, B)=\operatorname{Tr}\left[(A-B)^{2}\right]$ is the Hilbert-Schmidt distance.

Equation (19) shows that maximizing the sub-QFI $\mathcal{I}\left(\rho_{\theta}\right)$ is equivalent to maximizing the susceptibility of the Hilbert-Schmidt distance between the probe and exact states to changes in $\theta$, and hence, minimizing the super-fidelity. Here we recall that an equation similar to (19) can be obtained for the QFI by replacing the Hilbert-Schmidt distance by the Bures distance [53, 54], and for the Wigner-Yanase skew information in terms of the Hellinger distance [55].

Connection to the literature. Let us now discuss how the sub-QFI relates to other results in the literature, as this allows us to shed additional light on the properties of $\mathcal{I}(\rho)$. First, we note that the quantity $-\frac{1}{2} \operatorname{Tr}\left[[\rho, H]^{2}\right]$ was introduced in [21], as a lower-bound for the Wigner-Yanase skew information $I_{W Y}(\rho, H)=$ $-\frac{1}{2} \operatorname{Tr}\left[[\sqrt{\rho}, H]^{2}\right][56]$. Hence, we find

$$
I\left(\rho_{\theta}\right) \geqslant I_{W Y}(\rho, H) \geqslant \frac{\mathcal{I}\left(\rho_{\theta}\right)}{8} .
$$




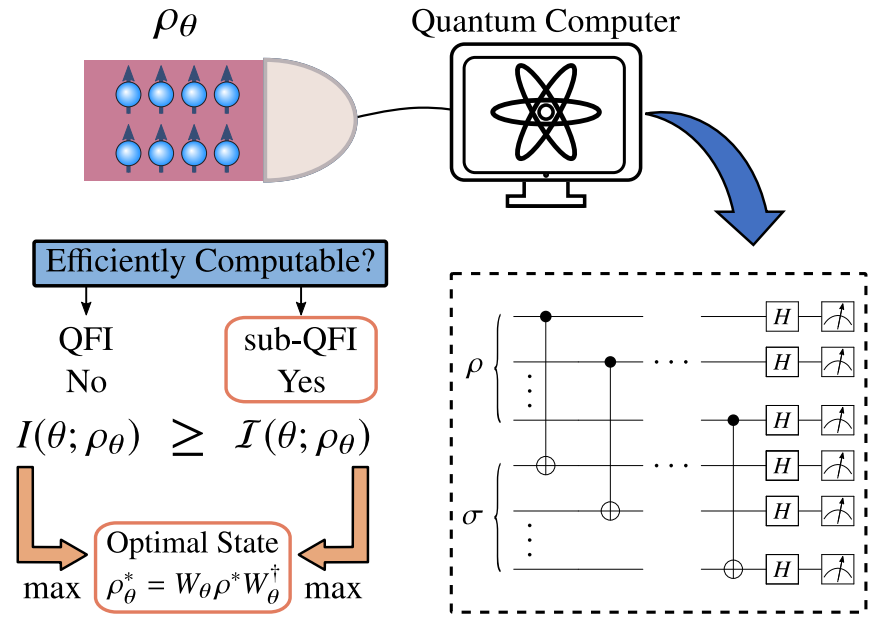

FIG. 2. Use of sub-QFI for quantum metrology. The sub-QFI can be efficiently computed from the exact state $\rho_{\theta}$ and error state $\rho_{\theta+\delta}$ via the depth-two circuit shown plus additional efficient classical post-processing [44] (here, $\rho=$ $\rho_{\theta}$ and $\left.\sigma=\rho_{\theta+\delta}\right)$. Since the QFI and sub-QFI share the same optimal input state $\rho^{*}$, the sub-QFI can be used as an efficiently computable surrogate for the QFI when preparing states for quantum metrology.

Since the Wigner-Yanase skew information is a measure of asymmetry [21, 57-59] and a quasi-measure of the $H$ coherence $[21,60,61]$ of the state $\rho$, then the sub-QFI can also be used as a bound for these quantities. We remark that in [62], the authors employ the quantity $-\frac{1}{2} \operatorname{Tr}\left[[\rho, H]^{2}\right]$ to derive a result similar to Proposition 2 .

The sub-QFI was also used in [45] to study of the relationship between quantum coherence and multiparticle entanglement [45]. Therein, the authors examine the relationship between multiple quantum coherence spectra (MQCs) and multiparticle entanglement witnesses. Specifically, it is shown that one can relate the MQCs to the QFI (a known entanglement witness) via the SubQFI in that the second moment of the MQC spectrum is equivalent to the Sub-QFI. Using the fact that the SubQFI inherits the property of being a witness of multiparticle entanglement from the QFI, they successfully establish the connection between MQCs and entanglement witnesses.

In addition, the sub-QFI can be directly related to a quantity known as the purity loss [22,34], which indicates the sensing performance of the probe state $\rho$. Specifically, the purity loss $\Delta \gamma$ quantifies the robustness of $\rho$ to statistical fluctuations in the parameter $\theta$. For instance, given a random variable $X$ normally distributed about $\theta$ with variance $(\Delta x)^{2}$, and which takes value $x$ with probability $\lambda_{x}$, one can define the ensemble-averaged state $\rho_{\text {ave }}=\sum_{x} \lambda_{x} \rho_{\theta(x)}\left(\right.$ with $\left.\rho_{\theta(x)}=W_{\theta(x)} \rho W_{\theta(x)}^{\dagger}\right)$. Since the purity of $\rho_{\text {ave }}$ is smaller than that of $\rho$, the purity loss is defined as

$$
\Delta \gamma=\operatorname{Tr}\left[\rho_{\theta}^{2}\right]-\operatorname{Tr}\left[\rho_{\text {ave }}^{2}\right] .
$$

Here, $\Delta \gamma$ quantifies the degree to which the probe state decoheres due to the statistical fluctuations in $\theta$ [22]. Similarly, the ratio $\Delta \gamma /(\Delta x)^{2}$ characterizes the fragility of $\rho$ to this stochastic noise.

From [22] we find that given a sharply distributed probability distribution of $X$ with variance $(\Delta x)^{2}$, the subQFI is related to the purity loss of (21) via

$$
\mathcal{I}\left(\rho_{\theta}\right) \approx 2 \frac{\Delta \gamma}{(\Delta x)^{2}}
$$

Equation (22) shows that the sub-QFI quantifies how fragile the probe state $\rho$ is to stochastic fluctuations in the parameter $\theta$.

Lastly, the sub-QFI has been explored in quantum optics applications [46, 47, 63]. Specifically, in [46] the authors derive the sub-QFI taking Eq. (19) in Proposition 2 as a starting point. Then, the authors explore the relevance of the sub-QFI in the context of quantum optics, showing that the sub-QFI can be used as a predictor of non-classicality in states of light $\rho$ under certain conditions. After defining the quantity $A_{H}=H^{2}-: H^{2}$ : (where :: denotes normal ordering of the operators), the authors show that for classical states the QFI satisfies $I(\rho) \leqslant \operatorname{Tr}\left[\rho A_{G}\right]$. Hence, $I(\rho)>\operatorname{Tr}\left[\rho A_{G}\right]$ implies that $\rho$ is non-classical, as long as a linear scheme is used [47]. One can then readily conclude that the sub-QFI serves as an efficiently computable indicator of non-classicality as $\mathcal{I}\left(\rho_{\theta}\right)>\operatorname{Tr}\left[\rho A_{G}\right]$ implies that $\rho$ is non-classical.

Sub-QFI for quantum metrology. The fact that the sub-QFI can be efficiently estimated in a quantum computer and that it is faithful to the QFI means it can be employed to variationally prepare optimal states for quantum metrology. Specifically, in this setting one trains a unitary $U$ acting on the input state with the goal of maximizing the sensing capabilities of $U \rho U^{\dagger}[33,36]$. As shown in Fig. 2, since one is unable to efficiently estimate the QFI, one instead computes the sub-QFI via the state overlap circuit [44]. Maximizing the sub-QFI means that one is able to guarantee a larger QFI value. While optimizing a lower bound could lead to a state being prepared that is not the optimal state for the QFI, Theorem 2 guarantees that the optimal state prepared by maximizing the sub-QFI will also maximize the QFI.

Conclusions. We have presented and analyzed the subQuantum Fisher Information (sub-QFI), an efficiently computable lower bound of quantum Fisher information (QFI). For unitary families, we have obtained rigorous results that allowed us to provide operational meaning the sub-QFI, including its connection to coherence and asymmetry measures, as well as its geometrical interpretation. In addition, we have proved that the sub-QFI is faithful to the QFI, in the sense that both quantities share the same global extrema.

Our results pave the way for the sub-QFI to be employed to prepare the optimal state in quantum metrology and quantum sensing applications. Moreover, due to the fact that the optimal state for sub-QFI can be deterministically prepared trough a variational circuit [36], 
preparing the sub-QFI can overcome some of the limitations that have been to arise for probabilistic state preparation [64], and hence thus enhancing the utility of sub-QFI.

We further remark that due to its connection to many quantities of interest, the sub-QFI plays an important role in bridging quantum metrology, quantum computing, and resource theories, and hence can help in advancing the understanding of the connection between these fields. Finally, we note that a future research direction is the study of the optimal measurement scheme associated with the optimal state for the sub-QFI.

\section{Acknowledgements}

We thank Davide Girolami and Lukas J. Fiderer for helpful discussions. This work was supported by the
Quantum Science Center (QSC), a National Quantum Information Science Research Center of the U.S. Department of Energy (DOE). MC and AS also acknowledge initial support from the Center for Nonlinear Studies at Los Alamos National Laboratory (LANL). AS is now supported by the internal R\&D from Aliro Technologies, Inc. JLB was initially supported by the U.S. DOE through a quantum computing program sponsored by the LANL Information Science \& Technology Institute. JLB was also supported by the National Science Foundation Graduate Research Fellowship under Grant No. 1650115. PJC also acknowledges initial support from the LANL ASC Beyond Moore's Law project.
[1] Dénes Petz and Catalin Ghinea, "Introduction to quantum fisher information," in Quantum probability and related topics (World Scientific, 2011) pp. 261-281.

[2] Masahito Hayashi, Quantum Information Theory: Mathematical Foundation (2nd edition) (Springer, 2004).

[3] Luca Pezzé and Augusto Smerzi, "Entanglement, nonlinear dynamics, and the Heisenberg limit," Phys. Rev. Lett. 102, 100401 (2009).

[4] Kavan Modi, Hugo Cable, Mark Williamson, and Vlatko Vedral, "Quantum correlations in mixed-state metrology," Phys. Rev. X 1, 021022 (2011).

[5] Akira Sone, Quntao Zhuang, and Paola Cappellaro, "Quantifying precision loss in local quantum thermometry via diagonal discord," Phys. Rev. A 98, 012115 (2018).

[6] Akira Sone, Quntao Zhuang, Changhao Li, Yi-Xiang Liu, and Paola Cappellaro, "Nonclassical correlations for quantum metrology in thermal equilibrium," Phys. Rev. A 99, 052318 (2019).

[7] Masahiro Takeoka and Mark M Wilde, "Optimal estimation and discrimination of excess noise in thermal and amplifier channels," arXiv preprint arXiv:1611.09165 (2016).

[8] Jonas Schuff, Lukas J Fiderer, and Daniel Braun, "Improving the dynamics of quantum sensors with reinforcement learning," New Journal of Physics 22, 035001 (2020).

[9] Vittorio Giovannetti, Seth Lloyd, and Lorenzo Maccone, "Advances in quantum metrology," Nat. Photonics 5, 222-229 (2011).

[10] Rafał Demkowicz-Dobrzański, Jan Kołodyński, and Mădălin Guţă, "The elusive Heisenberg limit in quantumenhanced metrology," Nature communications 3, 1-8 (2012).

[11] C. L. Degen, F. Reinhard, and P. Cappellaro, "Quantum sensing," Rev. Mod. Phys. 89, 035002 (2017).

[12] Luca Pezzè, Augusto Smerzi, Markus K. Oberthaler, Roman Schmied, and Philipp Treutlein, "Quantum metrology with nonclassical states of atomic ensembles," Rev. Mod. Phys. 90, 035005 (2018).
[13] Rosanna Nichols, Pietro Liuzzo-Scorpo, Paul A Knott, and Gerardo Adesso, "Multiparameter gaussian quantum metrology," Physical Review A 98, 012114 (2018).

[14] Vishal Katariya and Mark M Wilde, "Geometric distinguishability measures limit quantum channel estimation and discrimination," arXiv preprint arXiv:2004.10708 (2020).

[15] Teng-Long Wang, Ling-Na Wu, Wen Yang, Guang-Ri Jin, Neill Lambert, and Franco Nori, "Quantum fisher information as a signature of the superradiant quantum phase transition," New J. Phys. 16, 063039 (2014).

[16] En-Jia Ye, Zheng-Da Hu, and Wei Wu, "Scaling of quantum fisher information close to the quantum phase transition in the xy spin chain," Physica B: Condensed Matter 502, 151 (2016).

[17] Katarzyna Macieszczak, Mădălin Guţă, Igor Lesanovsky, and Juan P Garrahan, "Dynamical phase transitions as a resource for quantum enhanced metrology," Physical Review A 93, 022103 (2016).

[18] Joh Watrous, "Quantum statistical zero-knowledge," arXiv preprint arXiv:0202111 (0202111).

[19] Lukas J Fiderer, Tommaso Tufarelli, Samanta Piano, and Gerardo Adesso, "General expressions for the quantum fisher information matrix with applications to discrete quantum imaging," arXiv preprint arXiv:2012.01572 (2020).

[20] M. Cerezo, Alexander Poremba, Lukasz Cincio, and Patrick J Coles, "Variational quantum fidelity estimation," Quantum 4, 248 (2020).

[21] Davide Girolami, "Observable measure of quantum coherence in finite dimensional systems," Phys. Rev. Lett. 113, 170401 (2014).

[22] Kavan Modi, Lucas C. Céleri, Jayne Thompson, and Mile $\mathrm{Gu}$, "Fragile states are better for quantum metrology," arXiv preprint arXiv:1608.01443 (2016).

[23] Chao Zhang, Benjamin Yadin, Zhi-Bo Hou, Huan Cao, Bi-Heng Liu, Yun-Feng Huang, Reevu Maity, Vlatko Vedral, Chuan-Feng Li, Guang-Can Guo, and Davide Girolami, "Detecting metrologically useful asymmetry and entanglement by a few local measurements," Phys. Rev. A 
96, 042327 (2017).

[24] Geza Toth, "Lower bounds on the quantum fisher information based on the variance and various types of entropies," arXiv preprint arXiv:1701.07461 (2017).

[25] Iagoba Apellaniz, Matthias Kleinmann, Otfried Gühne, and Géza Tóth, "Optimal witnessing of the quantum fisher information with few measurements," Physical Review A 95, 032330 (2017).

[26] Akira Sone, M. Cerezo, Jacob L Beckey, and Patrick J Coles, "A generalized measure of quantum fisher information," arXiv preprint arXiv:2010.02904 (2020).

[27] John Preskill, "Quantum computing in the nisq era and beyond," Quantum 2, 79 (2018).

[28] M. Cerezo, Andrew Arrasmith, Ryan Babbush, Simon C Benjamin, Suguru Endo, Keisuke Fujii, Jarrod R McClean, Kosuke Mitarai, Xiao Yuan, Lukasz Cincio, and Patrick J. Coles, "Variational quantum algorithms," arXiv preprint arXiv:2012.09265 (2020).

[29] Suguru Endo, Zhenyu Cai, Simon C Benjamin, and Xiao Yuan, "Hybrid quantum-classical algorithms and quantum error mitigation," Journal of the Physical Society of Japan 90, 032001 (2021).

[30] Kishor Bharti, Alba Cervera-Lierta, Thi Ha Kyaw, Tobias Haug, Sumner Alperin-Lea, Abhinav Anand, Matthias Degroote, Hermanni Heimonen, Jakob S. Kottmann, Tim Menke, Wai-Keong Mok, Sukin Sim, Leong-Chuan Kwek, and Alán Aspuru-Guzik, "Noisy intermediate-scale quantum (nisq) algorithms," arXiv preprint arXiv:2101.08448 (2021).

[31] M. Cerezo, Kunal Sharma, Andrew Arrasmith, and Patrick J Coles, "Variational quantum state eigensolver," arXiv preprint arXiv:2004.01372 (2020).

[32] Carlos Bravo-Prieto, Ryan LaRose, M. Cerezo, Yigit Subasi, Lukasz Cincio, and Patrick Coles, "Variational quantum linear solver," arXiv preprint arXiv:1909.05820 (2019).

[33] Bálint Koczor, Suguru Endo, Tyson Jones, Yuichiro Matsuzaki, and Simon C Benjamin, "Variational-state quantum metrology," New Journal of Physics (2020), 10.1088/1367-2630/ab965e.

[34] Xiaodong Yang, Jayne Thompson, Ze Wu, Mile Gu, Xinhua Peng, and Jiangfeng Du, "Probe optimization for quantum metrology via closed-loop learning control," npj Quantum Inf 6, 62 (2020).

[35] Johannes Jakob Meyer, Johannes Borregaard, and Jens Eisert, "A variational toolbox for quantum multiparameter estimation," arXiv preprint arXiv:2006.06303 (2020).

[36] Jacob L Beckey, M. Cerezo, Akira Sone, and Patrick J Coles, "Variational quantum algorithm for estimating the quantum fisher information," arXiv preprint arXiv:2010.10488 (2020).

[37] Ziqi Ma, Pranav Gokhale, Tian-Xing Zheng, Sisi Zhou, Xiaofei Yu, Liang Jiang, Peter Maurer, and Frederic T Chong, "Adaptive circuit learning for quantum metrology," arXiv preprint arXiv:2010.08702 (2020).

[38] Samuel L Braunstein and Carlton M Caves, "Statistical distance and the geometry of quantum states," Physical Review Letters 72, 3439 (1994).

[39] Jing Liu, Heng-Na Xiong, Fei Song, and Xiaoguang Wang, "Fidelity susceptibility and quantum fisher information for density operators with arbitrary ranks," Physica A: Statistical Mechanics and its Applications 410, 167-173 (2014).
[40] Masahito Hayashi, Quantum Information Theory: Mathematical Foundation (2nd edition) (Springer, 2004).

[41] Jaroslaw Adam Miszczak, Zbigniew Puchala, Pawel Horodecki, Armin Uhlmann, and Karol Zyczkowski, "Sub- and super-fidelity as bounds for quantum fidelity," Quantum Information \& Computation 9, 103-130 (2009).

[42] Paulo EMF Mendonça, Reginaldo d J Napolitano, Marcelo A Marchiolli, Christopher J Foster, and YeongCherng Liang, "Alternative fidelity measure between quantum states," Physical Review A 78, 052330 (2008).

[43] Zbigniew Puchała and Jarosław Adam Miszczak, "Bound on trace distance based on superfidelity," Physical Review A 79, 024302 (2009).

[44] Lukasz Cincio, Yiğit Subaşı, Andrew T Sornborger, and Patrick J Coles, "Learning the quantum algorithm for state overlap," New Journal of Physics 20, 113022 (2018).

[45] Martin Gärttner, Philipp Hauke, and Ana Maria Rey, "Relating out-of-time-order correlations to entanglement via multiple-quantum coherences," Physical Review Letters 120, 040402 (2018).

[46] Ángel Rivas and Alfredo Luis, "Intrinsic metrological resolution as a distance measure and nonclassical light," Phys. Rev. A 77, 063813 (2008).

[47] Ángel Rivas and Alfredo Luis, "Precision quantum metrology and nonclassicality in linear and nonlinear detection schemes," Phys. Rev. Lett. 105, 010403 (2010).

[48] Shunlong Luo and Yuan Sun, "Skew information revisited: Its variants and a comparison of them," Theoretical and Mathematical Physics 202, 104-111 (2020).

[49] S. Alipour and A. T. Rezakhani, "Extended convexity of quantum fisher information in quantum metrology," Phys. Rev. A 91, 042104 (2015).

[50] Matteo GA Paris, "Quantum estimation for quantum technology," International Journal of Quantum Information 7, 125-137 (2009).

[51] Jing Liu, Jie Chen, Xiao-Xing Jing, and Xiaoguang Wang, "Quantum fisher information and symmetric logarithmic derivative via anti-commutators," Journal of Physics A: Mathematical and Theoretical 49, 275302 (2016).

[52] Lukas J Fiderer, Julien ME Fraïsse, and Daniel Braun, "Maximal quantum fisher information for mixed states," Physical Review Letters 123, 250502 (2019).

[53] Matthias Hübner, "Explicit computation of the bures distance for density matrices," Physics Letters. A 163, 239242 (1992).

[54] Dominik Šafránek, "Discontinuities of the quantum fisher information and the bures metric," Physical Review A 95, 052320 (2017).

[55] Shunlong Luo and Qiang Zhang, "Informational distance on quantum-state space," Physical Review A 69, 032106 (2004).

[56] Eugene P Wigner and Mutsuo M Yanase, "Information contents of distributions," in Part I: Particles and Fields. Part II: Foundations of Quantum Mechanics (Springer, 1997) pp. 452-460.

[57] Ryuji Takagi, "Skew informations from an operational view via resource theory of asymmetry," Scientific Reports 9, 1-12 (2019).

[58] Shunlong Luo and Yuan Sun, "Quantum coherence versus quantum uncertainty," Phys. Rev. A 96, 022130 (2017).

[59] Shunlong Luo, "Wigner-yanase skew information vs. quantum fisher information," Proceedings of the American Mathematical Society 132, 885-890 (2003). 
[60] Shuanping Du and Zhaofang Bai, "The wigner-yanase information can increase under phase sensitive incoherent operations," Annals of Physics 359, 136-140 (2015).

[61] Iman Marvian and Robert W Spekkens, "How to quantify coherence: Distinguishing speakable and unspeakable notions," Physical Review A 94, 052324 (2016).

[62] Benjamin Yadin and Vlatko Vedral, "General framework for quantum macroscopicity in terms of coherence," Phys- ical Review A 93, 022122 (2016).

[63] Andrei B Klimov, Marcin Zwierz, Sascha Wallentowitz, Marcin Jarzyna, and Konrad Banaszek, "Optimal lossy quantum interferometry in phase space," New Journal of Physics 19, 073013 (2017).

[64] Zdeněk Hradil, Jaroslav Řeháček, Luis Sánchez-Soto, and Berthold-Georg Englert, "Quantum fisher information with coherence," Optica 6, 1437 (2019). 


\section{Appendix}

Here we present the proof for the main results in our manuscript. For simplicity, we reiterate our theorems and propositions.

\section{Proof of Theorem 1}

Theorem 1 (Explicit form). For unitary families, the sub-QFI of Definition 1 can be expressed as

$$
\begin{aligned}
\mathcal{I}\left(\rho_{\theta}\right) & =4\left(\operatorname{Tr}\left[\rho^{2} H^{2}\right]-\operatorname{Tr}[\rho H \rho H]\right) \\
& =-2 \operatorname{Tr}\left[[\rho, H]^{2}\right] .
\end{aligned}
$$

Proof. First, recall that the super-fidelity defined as

$$
G(\rho, \sigma)=\operatorname{Tr}[\rho \sigma]+\sqrt{\left(1-\operatorname{Tr}\left[\rho^{2}\right]\right)\left(1-\operatorname{Tr}\left[\sigma^{2}\right]\right)} .
$$

In the case of unitary families, $\rho_{\theta}=W_{\theta} \rho W_{\theta}^{\dagger}$ with $W_{\theta}=e^{-i \theta H}$, and hence we have $\operatorname{Tr}\left[\rho^{2}\right]=\operatorname{Tr}\left[\rho_{\theta}^{2}\right]=\operatorname{Tr}\left[\rho_{\theta+\delta}^{2}\right](\operatorname{the}$ purity is unitarily invariant). Hence, the super fidelity between $\rho_{\theta}$ and $\rho_{\theta+\delta}$ can be simplified as

$$
G\left(\rho_{\theta}, \rho_{\theta+\delta}\right)=\operatorname{Tr}\left[\rho_{\theta} \rho_{\theta+\delta}\right]+1-\operatorname{Tr}\left[\rho^{2}\right]
$$

The first term can then be expressed as

$$
\begin{aligned}
\operatorname{Tr}\left[\rho_{\theta} \rho_{\theta+\delta}\right] & =\operatorname{Tr}\left[\left(W_{\theta} \rho W_{\theta}^{\dagger}\right)\left(W_{\theta} W_{\delta} \rho W_{\delta}^{\dagger} W_{\theta}^{\dagger}\right)\right] \\
& =\operatorname{Tr}\left[\rho e^{-i \delta H} \rho e^{+i \delta H}\right]
\end{aligned}
$$

where we have used the definition of the exact and error states and the cyclic property of the trace. Then, by employing the series expansion of $e^{-i \theta H}$ when $|\delta| \ll 1$

$$
e^{ \pm i \delta H}=\mathbb{1} \pm i \delta H-\frac{\delta^{2}}{2} H^{2}+\mathcal{O}\left(\delta^{3}\right)
$$

we can write

$$
\begin{aligned}
\operatorname{Tr}\left[\rho_{\theta} \rho_{\theta+\delta}\right] & =\operatorname{Tr}\left[\rho\left(\mathbb{1}-i \delta H-\frac{\delta^{2}}{2} H^{2}+\mathcal{O}\left(\delta^{3}\right)\right) \rho\left(\mathbb{1}+i \delta H-\frac{\delta^{2}}{2} H^{2}+\mathcal{O}\left(\delta^{3}\right)\right)\right] \\
& =\operatorname{Tr}\left[\rho^{2}+i \delta \rho^{2} H-\frac{\delta^{2}}{2} \rho^{2} H^{2}-i \delta \rho H \rho+\delta^{2} \rho H \rho H-\frac{\delta^{2}}{2} \rho H^{2} \rho+\mathcal{O}\left(\delta^{3}\right)\right] \\
& =\operatorname{Tr}\left[\rho^{2}\right]-\delta^{2}\left(\operatorname{Tr}\left[\rho^{2} H^{2}\right]-\operatorname{Tr}[\rho H \rho H]\right)+\mathcal{O}\left(\delta^{3}\right) .
\end{aligned}
$$

The super fidelity becomes

$$
\begin{aligned}
G\left(\rho_{\theta}, \rho_{\theta+\delta}\right) & =\operatorname{Tr}\left[\rho^{2}\right]-\delta^{2}\left(\operatorname{Tr}\left[\rho^{2} H^{2}\right]-\operatorname{Tr}[\rho H \rho H]\right)+\mathcal{O}\left(\delta^{3}\right)+1-\operatorname{Tr}\left[\rho^{2}\right] \\
& =1-\delta^{2}\left(\operatorname{Tr}\left[\rho^{2} H^{2}\right]-\operatorname{Tr}[\rho H \rho H]\right)+\mathcal{O}\left(\delta^{3}\right) .
\end{aligned}
$$

Expand the square root of $G\left(\rho_{\theta}, \rho_{\theta+\delta}\right)$ with respect to $\delta$, we find

$$
\sqrt{G\left(\rho_{\theta}, \rho_{\theta+\delta}\right)}=1-\frac{\delta^{2}}{2}\left(\operatorname{Tr}\left[\rho^{2} H^{2}\right]-\operatorname{Tr}[\rho H \rho H]\right)+\mathcal{O}\left(\delta^{3}\right),
$$

and rearranging we see from Eq. (5) that

$$
\mathcal{I}\left(\rho_{\theta}\right)=\lim _{\delta \rightarrow 0} 8 \frac{1-\sqrt{G\left(\rho_{\theta}, \rho_{\theta+\delta}\right)}}{\delta^{2}}=4\left(\operatorname{Tr}\left[\rho^{2} H^{2}\right]-\operatorname{Tr}[\rho H \rho H]\right) .
$$




\section{A. Proof of proposition 1}

Proposition 1 (Non-Hermitian SLD operator). For full rank probe states $\rho$, the sub-QFI can be expressed as

$$
\mathcal{I}\left(\rho_{\theta}\right)=2 \operatorname{Tr}\left[\Lambda_{\theta}^{\dagger} \Lambda_{\theta} \rho_{\theta}^{2}\right] .
$$

where $\Lambda_{\theta}$ is the $n S L D$ operator in (13).

Proof. For a full-rank probe state $\rho$, let us define $\Lambda_{\theta}$ as

$$
\Lambda_{\theta}=\left(\partial_{\theta} \rho_{\theta}\right) \rho_{\theta}^{-1}, \quad \Lambda_{\theta}^{\dagger}=\rho_{\theta}^{-1} \partial_{\theta} \rho_{\theta} .
$$

From Eq. (A2) we find that

$$
\begin{aligned}
\operatorname{Tr}\left[\Lambda_{\theta}^{\dagger} \Lambda_{\theta} \rho_{\theta}^{2}\right] & =\operatorname{Tr}\left[\left(\rho_{\theta}^{-1} \partial_{\theta} \rho_{\theta}\right)\left(\left(\partial_{\theta} \rho_{\theta}\right) \rho_{\theta}^{-1}\right) \rho_{\theta}^{2}\right] \\
& =\operatorname{Tr}\left[\left(\partial_{\theta} \rho_{\theta}\right)^{2}\right] \\
& =-\operatorname{Tr}\left[\left[\rho_{\theta}, H\right]^{2}\right] \\
& =-\operatorname{Tr}\left[[\rho, H]^{2}\right]
\end{aligned}
$$

where in Eq. (A5) we used the fact that since $\rho_{\theta}=W_{\theta} \rho W_{\theta}^{\dagger}$, then $\partial_{\theta} \rho_{\theta}=-i H W_{\theta} \rho W_{\theta}^{\dagger}+i W_{\theta} \rho W_{\theta}^{\dagger} H=i\left[\rho_{\theta}, H\right]$. Then, Eq. (A6) is obtained by noting that $\operatorname{Tr}\left[\left[\rho_{\theta}, H\right]\right]=[\rho, H]$ since $\left[W_{\theta}, H\right]=0$. Finally, combining the definition of the sub-QFI in Eq. (11) with (A6), we prove Proposition 1.

Here, note that for full-rank states, the QFI can be expressed as $I\left(\rho_{\theta}\right)=\operatorname{Tr}\left[\Lambda_{\theta}^{2} \rho_{\theta}\right]$ when $\left[\rho_{\theta}, \partial_{\theta} \rho_{\theta}\right]=0$. In this case, $\Lambda_{\theta}$ becomes the SLD operator [51]

$$
\Lambda_{\theta}=L_{\theta}=\rho_{\theta}^{-1} \partial_{\theta} \rho_{\theta}=\left(\partial_{\theta} \rho_{\theta}\right) \rho_{\theta}^{-1},
$$

which matches to Eq. (2).

\section{B. Proof of Theorem 2}

Theorem 2 (Optimal state). For any quantum state $\rho$ and for any Hermitian generator H, the sub-QFI of Definition 1 and the QFI in (4) are maximized for the same state preparation $U \rho U^{\dagger}$, with the optimal unitary being

$$
U_{*}=\underset{U}{\arg \max } \mathcal{I}\left(U \rho U^{\dagger}\right)=\underset{U}{\arg \max } I\left(U \rho U^{\dagger}\right) .
$$

Here, the maximum is taken over the unitary group of degree $d$, with $d=2^{n}$.

Let us remark that the proof employed here follows the proof in Ref. [52] for determining the optimal mixed state for the QFI. While the main steps in the proof are the same, we nevertheless present here the derivation of the state that maximizes sub-QFI. Moreover, for convenience of the reader, we present additional details in the proof that clarify the steps followed.

The main idea of the proof is to construct an upper bound, $\mathcal{J}(\rho) \geqslant \mathcal{I}(\rho)$, for the sub-QFI $\mathcal{I}(\rho)$, that has simpler behavior than the sub-QFI itself. Note that for simplicity of notation we drop the $\theta$ dependence of $\mathcal{I}$. the If one can find the state, denoted $\rho^{*}$, that maximizes this upper bound and then show that $\mathcal{J}\left(\rho^{*}\right)=\mathcal{I}\left(\rho^{*}\right)$, one will have shown that $\rho^{*}$ maximizes the sub-QFI, as desired.

First, recall from Theorem 1, that the sub-QFI can be expressed as

$$
\mathcal{I}(\rho)=4\left(\operatorname{Tr}\left[\rho^{2} H^{2}\right]-\operatorname{Tr}[\rho H \rho H]\right) .
$$

Let $\{|k\rangle\}_{k=1}^{d}$ denote the eigenbasis for $\rho$, so that

$$
\rho=\sum_{k=1}^{d} \lambda_{k}|k\rangle\langle k| .
$$


Then, the sub-QFI can be expressed as

$$
\mathcal{I}(\rho)=\frac{1}{2} \sum_{i, j=1}^{d}\left(\lambda_{i}-\lambda_{j}\right)^{2}\left|h_{i j}\right|^{2},
$$

where $h_{i j}=\langle i|H| j\rangle$ are the matrix elements of $H$ in the eigenbasis of $\rho$, and where we have symmetrized the result to put indices $i, j$ on equal footing. Now, let us define

$$
\lambda_{i j}= \begin{cases}0 & \text { if } \lambda_{i}=\lambda_{j}=0, \\ \left(\lambda_{i}-\lambda_{j}\right)^{2} & \text { else. }\end{cases}
$$

As noted in the main text, the sub-QFI and QFI have very similar explicit forms. The only difference is in the form of the coefficients in their explicit representations. It is this observation that makes our problem of finding the optimal state for the sub-QFI amenable to the techniques used in Ref. [52]. Now that we have defined the coefficients $\lambda_{i j}$, we prove some essential inequalities for them.

Lemma 1. Let $\lambda_{1} \geqslant \ldots \geqslant \lambda_{d} \geqslant 0$. Then the following inequalities hold:

$$
\begin{aligned}
& \text { 1. } \lambda_{i, l} \geqslant \lambda_{i, j}+\lambda_{j, l} \text { for } 1 \leqslant i<j<l \leqslant d \text {, } \\
& \text { 2. } \lambda_{i, l}-\lambda_{i+1, l} \geqslant \lambda_{i, k}-\lambda_{i+1, k} \text { for } 1<i+1<k<l \leqslant d \\
& \text { 3. } \lambda_{i, l}-\lambda_{i, l-1} \geqslant \lambda_{j, l}-\lambda_{j, l-1} \text { for } 1 \leqslant i<j<l-1<d
\end{aligned}
$$

Proof. The three items in the above lemma are special cases of the following inequality:

$$
\lambda_{i, l}+\lambda_{j, k} \geqslant \lambda_{i, k}+\lambda_{j, l} \text { for } 1 \leqslant i<j<k<l \leqslant d .
$$

To show that (B6) holds, we note that $1 \leqslant i<j<k<l \leqslant d \Longrightarrow \lambda_{d} \leqslant \lambda_{l}<\lambda_{k}<\lambda_{j}<\lambda_{i} \leqslant 1$, and observe

$$
\begin{aligned}
\lambda_{i, l}+\lambda_{j, k}-\lambda_{i, k}-\lambda_{j, l} & =\left(\lambda_{i}-\lambda_{l}\right)^{2}+\left(\lambda_{j}-\lambda_{k}\right)^{2}-\left(\lambda_{i}-\lambda_{k}\right)^{2}-\left(\lambda_{j}-\lambda_{l}\right)^{2} \\
& =2\left[\lambda_{i}\left(\lambda_{k}-\lambda_{l}\right)-\lambda_{j}\left(\lambda_{k}-\lambda_{l}\right)\right] \\
& \geqslant 0 .
\end{aligned}
$$

For the first inequality above, we let $j=k$. For the second and third inequalities, we let $j=i+1$ and $k=l-1$, respectively. Then, the final inequality follows from the fact that $\lambda_{i}>\lambda_{j}$.

A crucial step in constructing our upper bound is introducing new coefficients defined in terms of the $\lambda_{i, j}$ 's as

$$
q_{i, j}:=\sum_{k=i}^{j-1} q_{k, k+1},
$$

for $i<j$. These coefficients play a crucial role in the proof of the optimal state due to the following lemma.

Lemma 2. For any dimension $d \geqslant 2$ and any ordered, non-negative coefficients $\lambda_{1} \geqslant \ldots \lambda_{d} \geqslant 0$, there exist coefficients $q_{k, k+1} \geqslant 0$ with $1 \leqslant k \leqslant d-1$ such that for $1 \leqslant i<j \leqslant d$ :

$$
\begin{array}{ll}
q_{i, j}=\lambda_{i, j} & \text { if } j=d-i+1, \\
q_{i, j} \geqslant \lambda_{i, j} & \text { else. }
\end{array}
$$

Here, although the form of the coefficients in the explicit representation of sub-QFI differ from the QFI, the proof of this proposition is identical to Ref. [52], so we proceed to the proof of Theorem 2.

Proof. As previously mentioned, we will first show that there exists an our upper bound, $\mathcal{J}(\rho) \geqslant \mathcal{I}(\rho)$, for the sub-QFI $\mathcal{I}(\rho)$ which is maximized by some $\rho^{*}$. We then show that $\mathcal{J}\left(\rho^{*}\right)=\mathcal{I}\left(\rho^{*}\right)$, which implies that $\rho^{*}$ maximizes the subQFI, as desired. Finally, by inspection, one will be able to see that the state that maximizes the sub-QFI is identical to the state that maximizes the QFI from Ref. [52]. Specifically, if we let $\left\{h_{j}\right\}$ and $\left\{\left|h_{j}\right\rangle\right\}$ respectively be the set of 
eigenvalues and associated eigenvectors of $H$ (with $\left.h_{j} \geqslant h_{j+1}\right)$. Then, as shown in [52] the state that maximizes the QFI, and hence the sub-QFI, is given by $\rho^{*}=\sum_{j=1}^{d} \lambda_{j}\left|\phi_{j}\right\rangle\left\langle\phi_{j}\right|$, where

$$
\left|\phi_{j}\right\rangle= \begin{cases}\frac{1}{\sqrt{2}}\left|h_{j}\right\rangle+\frac{1}{\sqrt{2}} e^{i \chi}\left|h_{d-j+1}\right\rangle & (2 j<d+1) \\ \left|h_{j}\right\rangle & (2 j=d+1) \\ \frac{1}{\sqrt{2}}\left|h_{j}\right\rangle-\frac{1}{\sqrt{2}} e^{i \chi}\left|h_{d-j+1}\right\rangle & (2 j>d+1)\end{cases}
$$

and where $\chi \in \mathbb{R}$ is an arbitrary phase.

Unitary transformations of the form, $U \rho U^{\dagger}$ leave the eigenvalues of $\rho$ unchanged. This unitary freedom allows one to change between ordered orthonormal bases. As such, optimizing over unitary preparations is equivalent to optimizing over ordered bases $B \in S$ with

$$
S:=\left\{\left|e_{i}\right\rangle:\left\langle e_{i} \mid e_{j}\right\rangle=\delta_{i, j} \quad \forall i, j \in\{1, \ldots, d\}\right\}
$$

The only reason one is restricted to ordered bases is because the ordered eigenvectors correspond to an ordered set of associated eigenvalues which is crucial for the result.

Let us denote the optimal ordered basis as $B^{*}=\left\{\left|\phi_{i}\right\rangle\right\}_{i=1}^{d}$. Then, the maximization problem is solved by

$$
\mathcal{I}\left(\rho^{*}\right):=\max _{B \in S} \mathcal{I}(B)
$$

where we have simply expressed the sub-QFI as a function of the ordered basis, $B$. That is,

$$
\begin{aligned}
\mathcal{I}(B) & =2 \sum_{i, j=1}^{d} \lambda_{i, j}\left|h_{i, j}(B)\right|^{2} \\
& =4 \sum_{i=1}^{d-1} \sum_{j=i+1}^{d} \lambda_{i, j}\left|h_{i, j}(B)\right|^{2}
\end{aligned}
$$

where $h_{i, j}=\left\langle e_{i}|H| e_{j}\right\rangle$ are the matrix elements of the generator with respect to the ordered basis $B=\left\{|e\rangle_{i}\right\}_{i=1}^{d}$. We remark that $\lambda_{i, j}=\lambda_{j, i}$ and that $\left|h_{i, j}(B)\right|^{2}=\left|h_{j, i}(B)\right|^{2}$, as these facts will be used in what follows.

To obtain the upper bound $\mathcal{J}(B)$ on the sub-QFI, we replace the $\lambda_{i, j}$ coefficients with the $q_{i, j}$ coefficients from Eq. (B10). That is, defining

$$
\mathcal{J}(B):=4 \sum_{i=1}^{d-1} \sum_{j=i+1}^{d} q_{i, j}\left|h_{i, j}(B)\right|^{2},
$$

we readily find from Lemma 2 that

$$
\mathcal{I}(B) \leqslant \mathcal{J}(B)
$$

Next, using the fact that $q_{i, j}=\sum_{k=i}^{j-1} q_{k, k+1}$ for all $1 \leqslant i<j \leqslant d$, we can write

$$
\begin{aligned}
\mathcal{J}(B) & =4 \sum_{i=1}^{d-1} \sum_{j=i+1}^{d} q_{i, j}\left|h_{i, j}(B)\right|^{2} \\
& =4 \sum_{i=1}^{d-1} \sum_{j=i+1}^{d}\left(\sum_{k=i}^{j-1} q_{k, k+1}\right)\left|h_{i, j}(B)\right|^{2} \\
& =4 \sum_{k=1}^{d-1} q_{k, k+1}\left(\sum_{i=1}^{k} \sum_{j=k+1}^{d}\left|h_{i, j}(B)\right|^{2}\right) .
\end{aligned}
$$

Now, to simplify further, we rewrite the term in the parenthesis as a Hilbert-Schmidt norm. Recalling that the Hilbert-Schmidt norm is defined as $\|A\|_{2}^{2}:=\sum_{i=1}^{m} \sum_{j=1}^{n}\left|A_{i, j}\right|^{2}$, one we can identify the parenthetical term as the 
Hilbert-Schmidt norm of the subblock of $H$ spanning from the 1 st to $k$-th row and from the $(k+1)$-th to the $d$-th column. With this insight, one can write

$$
\mathcal{J}(B)=4 \sum_{k=1}^{d-1} q_{k, k+1}\|h(B, k)\|_{2}^{2},
$$

where as in Ref. [52] we denote the aforementioned subblock as $h(B, k)$.

Expressing the upper bound in terms of Hilbert-Schmidt norms allows us to use the Bloomfield-Watson inequality, which bounds the norm of off-diagonal blocks of matrices. The relevant form of the inequality is

$$
\|h(B, k)\|_{2}^{2} \leqslant \frac{1}{4} \sum_{i=1}^{m(k)}\left(h_{i}-h_{d-i+1}\right)^{2}
$$

where $m(k)=\min \{k, d-k\}$. If the conjectured optimal basis $\left\{\left|\phi_{i}\right\rangle\right\}_{i=1}^{d}$ of Eq. (B13) is optimal, then the left-hand side of the Bloomfield-Watson inequality is saturated. Explicitly, we can write

$$
\begin{aligned}
\left\|h\left(B^{*}, k\right)\right\|_{2}^{2} & =\sum_{i=1}^{k} \sum_{j=k+1}^{d}\left|h_{i, j}\left(B^{*}\right)\right|^{2} \\
& =\sum_{i=1}^{k} \sum_{j=k+1}^{d}\left|\left\langle\phi_{i}|H| \phi_{j}\right\rangle\right|^{2} .
\end{aligned}
$$

Then, from Eq. (16) we see that the form of the optimal eigenvectors is different when $2 k<d+1,2 k=d+1$, or $2 k>d+1$. When $i=1$, we see that $\left|\phi_{1}\right\rangle$ contains both $\left|h_{1}\right\rangle$ and $\left|h_{d}\right\rangle$. Then for $\left|\phi_{2}\right\rangle$ we will have both $\left|h_{2}\right\rangle$ and $\left|h_{d-1}\right\rangle$, and so on. Hence, computing the overlaps $\left\langle\phi_{i}|H| \phi_{j}\right\rangle$ will yield delta functions of the form $\delta_{i, j}$ (prohibited from the fact that the sum is over off-diagonal terms) and $\delta_{i, d-j+1}$. Then, we are left with

$$
\begin{aligned}
\left\|h\left(B^{*}, k\right)\right\|_{2}^{2} & =\sum_{i=1}^{k} \sum_{j=k+1}^{d}\left|\left\langle\phi_{i}|H| \phi_{j}\right\rangle\right|^{2} \\
& =\sum_{i=1}^{k} \sum_{j=k+1}^{d}\left(\delta_{i, d-j+1} \frac{\left(h_{i}-h_{d-i+1}\right)}{2}\right)^{2} \\
& =\frac{1}{4} \sum_{i=1}^{m(k)}\left(h_{i}-h_{d-i+1}\right)^{2}
\end{aligned}
$$

where the final sum over $i$ ranges from 1 to $m(k)=\min \{k, d-k\}$. This is to avoid double counting in the case where $k>d / 2$. Comparing Eq. (B29) to Eq. (B24), we see that the Bloomfield-Watson inequality is in fact saturated when $B=B^{*}$. The Hilbert-Schmidt norm of the off-diagonal blocks of the generator are thus maximized for $B=B^{*}$ meaning $\|h(B, k)\|_{2}^{2} \leqslant\left\|h\left(B^{*}, k\right)\right\|_{2}^{2}$ which implies

$$
\mathcal{J}(B) \leqslant \mathcal{J}\left(B^{*}\right) \quad \forall B \in S
$$

Now that we have established that $B^{*}$ maximizes the upper bound on the QFI, it remains to be shown that $\mathcal{J}\left(B^{*}\right)=\mathcal{I}\left(B^{*}\right)$. From the definition of $\mathcal{J}(B)$, we have

$$
\begin{aligned}
\mathcal{J}\left(B^{*}\right) & =4 \sum_{i=1}^{d-1} \sum_{j=i+1}^{d} q_{i, j}\left|h_{i, j}\left(B^{*}\right)\right|^{2} \\
& =4 \sum_{i=1}^{d-1} \sum_{j=i+1}^{d} q_{i, j}\left(\delta_{i, d-j+1} \frac{h_{i}-h_{d-i+1}}{2}\right)^{2} \\
& =\frac{1}{2} \sum_{i=1}^{d} \lambda_{i, d-i+1}\left(h_{i}-h_{d-i+1}\right)^{2} \\
\mathcal{J}\left(B^{*}\right) & =\mathcal{I}\left(B^{*}\right)
\end{aligned}
$$


where we have used Lemma 1 to assert $q_{i, d-i+1}=\lambda_{i, d-i+1}$ and where we revert to a sum from $i=1$ to $i=d$ which requires a factor of $1 / 2$ to avoid double counting. We have now established that the Sub-QFI and QFI are maximized by the same state. This is equivalent to saying they are maximized by the same unitary state preparation. Thus, the Theorem 2 from the main text has been proven.

\section{Proof of Proposition 2}

Proposition 2 (Geometrical interpretation). The sub-QFI can be expressed as

$$
\mathcal{I}\left(\rho_{\theta}\right)=\left.\partial_{\theta}^{2} D_{H S}\left(\rho, \rho_{\theta}\right)\right|_{\theta=0},
$$

where $D_{H S}(A, B)=\operatorname{Tr}\left[(A-B)^{2}\right]$ is the Hilbert-Schmidt distance.

Proof.

$$
\begin{aligned}
\partial_{\theta}^{2} D_{H S}\left(\rho, \rho_{\theta}\right) & =\partial_{\theta}^{2}\left(\operatorname{Tr}\left[\left(\rho-\rho_{\theta}\right)^{2}\right]\right), \quad\left(\text { definition of } D_{H S}\left(\rho, \rho_{\theta}\right)\right) \\
& =\partial_{\theta}^{2}\left(\operatorname{Tr}\left[\rho^{2}-\rho \rho_{\theta}-\rho_{\theta} \rho+\rho_{\theta}^{2}\right]\right), \\
& =2 \partial_{\theta}^{2}\left(\operatorname{Tr}\left[\rho^{2}\right]-\operatorname{Tr}\left[\rho \rho_{\theta}\right]\right), \quad(\text { linearity and cyclicity of trace }) \\
& =-2 \operatorname{Tr}\left[\rho \partial_{\theta}^{2} \rho_{\theta}\right] . \quad\left(\partial_{\theta} \operatorname{Tr}\left[\rho^{2}\right]=0\right)
\end{aligned}
$$

The first derivative of the exact state with respect to $\theta$ can be shown to be $\partial_{\theta} \rho_{\theta}=i e^{-i \theta H}[\rho, H] e^{+i \theta H}$. The second derivative is then

$$
\begin{aligned}
\partial_{\theta}^{2} \rho_{\theta} & =\partial_{\theta}\left[i e^{-i \theta H}[\rho, H] e^{+i \theta H}\right], \\
& =H e^{-i \theta H}[\rho, H] e^{i \theta H}-e^{-i \theta H}[\rho, H] H e^{i \theta H} .
\end{aligned}
$$

So, substituting back into Eq. (C5), we have

$$
\begin{aligned}
\partial_{\theta}^{2} D_{H S}\left(\rho, \rho_{\theta}\right) & =-2 \operatorname{Tr}\left[\rho\left(H e^{-i \theta H}[\rho, H] e^{i \theta H}-e^{-i \theta H}[\rho, H] H e^{i \theta H}\right)\right], \\
& =-2 \operatorname{Tr}\left[\rho H \rho_{\theta} H-\rho H H \rho_{\theta}-H \rho \rho_{\theta} H+H \rho H \rho_{\theta}\right],
\end{aligned}
$$

where we have used $\left[H, e^{ \pm i \theta H}\right]=0$, and the linearity and cyclicity of trace. Now, letting $\theta \rightarrow 0$ we arrive at the final expression

$$
\lim _{\theta \rightarrow 0} \partial_{\theta}^{2} D_{H S}\left(\rho, \rho_{\theta}\right)=-2 \operatorname{Tr}\left[[\rho, H]^{2}\right] .
$$

Comparing to Eq. (11), we see that

$$
\mathcal{I}\left(\rho_{\theta}\right)=\left.\partial_{\theta}^{2} D_{H S}\left(\rho_{\theta}\right)\right|_{\theta=0} .
$$

